$$
\text { DOE/ER/60673-. T/3 }
$$

\title{
NEUTRON SCATTER STUDIES OF CHROMATIN STRUCTURE RELATED TO FUNCTIONS
}

\author{
DE-FG03 88ER60673 A000 \\ PROGRESS REPORT (7/1/88-6/30/89)
}

DOE/ER/ $60673--T 3$

DE92 011630

\section{Neutron Scatter Studies}

Neutron scatter studies have been performed at LANSCE, LANL and at the Institute Laue Langevin, Grenoble, France. In the previous progress report $(4 / 1 / 88-7 / 1 / 88)$ the following objectives were listed:

i) Shape of the histone octamer.

ii) Location of the $\mathrm{N}$-terminal domains of histone in the nucleosome core particle (specific aim 1 of original grant proposal).

iii) Effect of acetyiation on nucleosome structure (specific aim 2).

iv) Location of the globular domain of histone HI (specific aim 6).

v) Complexes of the transcription factor IIIA with its DNA binding site.

i) Shape of the histone octamer (work completed, manuscript in preparation).

There is a controversy concerning the shape of the histone octamer. In the crystal structure of the nucleosome core particle the observed electron density of the histone octamer, which is $85 \%$ of the expected electron density, occupies a flat cylindrical shape $7 \mathrm{~nm}$ diameter by $5.5 \mathrm{~nm}$ thick. Its calculated radius of gyration $(\mathrm{Rg})$ is $2.94 \mathrm{~nm}$. The crystal structure of the isolated octamer is different and has a shape more like a rugby ball, $11 \mathrm{~nm} \times 6.5 \mathrm{~nm} \times 7.0 \mathrm{~nm}$ with a calculated $\mathrm{Rg}$ of 3.3 to $3.4 \mathrm{~nm}$.

To determine whether there is histone octamer polymorphism, we have carried out neutron and $\mathrm{x}$-ray scatter studies of isolated histone octamers in different salt conditions: $2.0 \mathrm{M}$ $\mathrm{NaCl}$ and $2.3 \mathrm{M}\left(\mathrm{NH}_{4}\right)_{2} \mathrm{SO}_{4}$. The values we obtain are given in the following table.

\section{Neutron Scatter Radius of Gyration}

Core particle in $0.1 \mathrm{M} \mathrm{NaCl}$ at $65 \%{ }^{2} \mathrm{H}_{2} \mathrm{O}: 37 \%{ }^{1} \mathrm{H}_{2} \mathrm{O}$

Core particle dissociated in $2.0 \mathrm{MNaCl}$ at $65 \%{ }^{2} \mathrm{H}_{2} \mathrm{O}: 37 \%{ }^{1} \mathrm{H}_{2} \mathrm{O}$

Isolated octamer in $2.0 \mathrm{MNaCl}$

Isolated octamer in $2.2 \mathrm{M}\left(\mathrm{NH}_{4}\right)_{2} \mathrm{SO}_{4}$
$3.30 \mathrm{~nm}$

$3.17 \mathrm{~nm}$

3.13-3.30 nm

3.4-3.48 nm

These results provide evidence for histone octamer polymorphism and a model that is based on the behaviors of th: $N$-terminal domains of the histone provides an explanation for the octamer polymorphisms. A manuscript describing this work will be completed and submitted for publication by the end of October.

ii) Location of histone $\mathrm{N}$-terminal domains in histone ectamers and nucleosome core

The $\mathrm{N}$-terminal domains of the core histone octamer that contain all of the sites of reversible acety!ations required for such DNA functions as replication and transcription ar thought to interact with the DNA regions entering and leaving the nucleosome. Thus

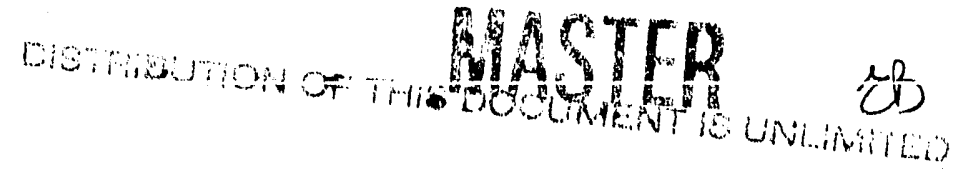


modulation of these interactions through reversible chemical modifications would be expected to affect the packaging of nucleosomes into higher order chromatin structures.

Neutron scatter studies of the nucleosome core particle at the DNA contrast matched position give an $\mathrm{Rg}$ for the histone octamer of $3.3 \mathrm{~nm}$. To account for the difference between this measured $\mathrm{Rg}$ of $3.3 \mathrm{~nm}$ and the calculated $\mathrm{Rg}$ of $2.94 \mathrm{~nm}$ for the observed partial electron density of the octamer in the core particle, we have proposed that the octamer histone Nterminal domains are disordered in the core particle crystal structure. To test this proposal we have carried out two experiments; a) the removal of the $\mathrm{N}$-terminal domains from the histone octamer by controlled proteolysis and determination of the radius of gyration of the resulting "trimmed" octamer and b) removal of the $\mathrm{N}$-terminal domains from the nucleosome core particle and determination of the neutron scatter fundamental scatter curves for the "trimmed" core particle.

a) Neutron scatter studies of the "trimmed" histone octamer. From the neutron scatter studies the radius of gyration of the "trimmed" octamer is $2.98 \mathrm{~nm}$ identical to the calculated value for the histone octamer $\mathbf{R g}$ of $2.94 \mathrm{~nm}$ in the core particle crystal structure. This result suggests that an understanding of the behaviors of these $\mathrm{N}$-terminal "functional" domains will provide an explanation for the published "different" shapes for the histone octamer in the core particle and isolated octamer crystals.

b) Neutron scatter of the "trimmed' core particle. A full neutron scatter contrast matching experiment on the "trimmed" core particle has been carried out at the Institute Laue Langevin. The fundamental scatter functions $I_{C}\left(\right.$ shape), Is (internal structure) and $I_{C s}$ have been obtained and compared with the same functions from the control core particles. Whereas these functions for the control core particle are best fitted to a flat disc $11.0 \mathrm{~nm} \times 5.5-6.0 \mathrm{~nm}$, the functions obtained from the "trimmed" core particle are sufficiently different to suggest a structural transition to a more globular shape. This would be the first evidence for a different shape for the nucleosome core particle on removal of the $\mathrm{N}$-terminal domains. It is therefore necessary to repeat this experiment to confirm these unexpected results and to obtain a low resolution structure for the "trimmed" core particle.

\section{Neutron scatter experiments at LANSCE, Los Alamos National Lab.}

Six days of neutron scatter time were allocated at LANSCE for three experiments:

I) Solution structure of the transcription facter IIIA/DNA complex. Transcription factor IIIA is a member of a major ciass of DNA regulatory proteins. It binds to a regulatory sequence that is internal to the 5S ribosomal RNA gene and binds also to the RNA gene product. Because of this ability to bind both to the gene and its RNA product, it has been proposed that transcription factor IIIA represents an early protein in evolution. This class of DNA regulatory proteins is characterized by a protein binding motif called a zinc finger and these proteins are described as "zinc finger proteins." Zinc fingers contain 30 amino acids with 4 cysteines and histidines at the base of the finger providing a zinc binding site. In TFIIIA there are 9 consecutive zinc fingers that bind to a specific 59 bp DNA sequence. Two models have been proposed by A. Klug's group in Cambridge, UK. In one model the TFIIIA lies along one side of a linear DNA molecule with the consecutive zinc fingers protruding into the consecutive DNA grooves to recognize the DNA sequence. In the second model TFIIIA winds itself around its DNA binding site in the deep groove of a linear DNA molecule. Because of the central importance of this class of DNA regulating proteins in cell and molecular biology we carried out two experiments to test the proposed models: i) the effect of TFIIIA binding on DNA bending and ii) a partial neutron scatter study of the TFIIIA/DNA complex at LANSCE. 
i) Effect of the binding of TEIIIA on DNA bending (Schroth et al., 1989 Nature 340, 487-488). If the path of the specific DNA sequence is unaffected by the binding of the TFIIIA then there should be no effect of TFIIIA on the TFIIIA DNA bandshift in the circular permutation and circularization assay. As described in the attached letter to Nature, this was not the case and convincing evidence was presented showing that a major bend was induced in the specific DNA site by the binding of TFIIIA.

ii) Neutron scatter studies of TFIIIA/DNA comolexes. The initial proposal was to use neutron techniques to distinguish between the two models for TFIIIA/DNA complexes proposed by Klug as outlined above i.e. TFIIIA binding on one side of its DNA binding site or winding around the DNA in the deep groove. For both models the $R_{g}$ of the DNA would be about $5.0 \mathrm{~nm}$ whereas the $\mathrm{Rg}$ of the TFIIIA would be quite different for each model. Two days of neutron scatter time were awarded for this experiments at LANSCE to carry out a neutron scatter contrast matching experiment on the complex. Such a study gives the shape of the complex, the spatial organization of the DNA and protein components and the Rgs of the protein and DNA components. To obtain all this structure information, four neutron scatter measurements have to be made; i) $100 \%{ }^{2} \mathrm{H}_{2} \mathrm{O}$; ii) $63 \%{ }^{2} \mathrm{H}_{2} \mathrm{O}: 37 \%{ }^{1} \mathrm{H}_{2} \mathrm{O}$ (DNA match point); iii) $40 \%{ }^{2} \mathrm{H}_{2} \mathrm{O}: 60 \%{ }^{1} \mathrm{H}_{2} \mathrm{O}$ (protein match point) and iv) $100{ }^{1} \mathrm{H}_{2} \mathrm{O}$. Unfortunately, because of a breakdown in the delivery of the proton beam to the neutron spallation source only the first measurement could be carried out. From this measurement we obtained an $\mathrm{Rg}_{\mathrm{g}}$ for the TFIIIA/DNA complex in $100 \%{ }^{2} \mathrm{H}_{2} \mathrm{O}$ of $37 \mathrm{~A}$ indicating that the DNA is bent. This experiment has to be completed.

The above experiments show that both the previous proposed model were incorrect and models are being developed that incorporate the fact that the DNA binding site undergoes a major bend on the binding of TFIIIA.

II) Effect of histone acetylation on nucleosome structure (manuscript completed by Marvin, K.M. and Bradbury, E.M. "Reconstitution of fully acetylated nucleosomes from defined components.")

The attached earlier reprint [Appendix I] describes a neutron scatter study of a hyperacetylated nucleosome core particle. No effect of acetylation was observed on the low resolution structure of the core particle. The core particle, however, is a subnucleosome particle that contains $146 \mathrm{bp}$ DNA compared to the 170 to $212 \mathrm{bp}$ of DNA found in nucleosomes. If the effects of histone acetylation were on the DNA regions entering and leaving the nucleosome, then no effect of acetylation would be observed on the core particle because these DNA regions have been removed. To study the effect of histone acetylation on the structure of the nucleosome, we have taken the approach of reforming nucleosomes fully defined with respect to DNA sequence, DNA length and states of histone acetylation. A manuscript describing this procedure has been completed. The precise DNA sequence comes from the 5S RNA gene and contains both a nucleosome locating sequence and a TFIIIA binding site. The DNA lengths we are working with at this time are 172,195 and $207 \mathrm{bp}$. To determine the effect of histone acetylation at the nucleosome level we have first to carry out a neutron scatter study of the control non-acetylated nuclesome.

Neutron scatter study of a reformed 195 bo DNA nucleosome. Two days of nedtron time were awarded at LANSCE for this experiment. Measurements were made at 4 contrasts; $100 \%{ }^{2} \mathrm{H}_{2} \mathrm{O} ; 63 \%$ 2; 63\% ${ }^{2} \mathrm{H}_{2} \mathrm{O}: 37 \%{ }^{1} \mathrm{H}_{2} \mathrm{O} ; 40 \%{ }^{2} \mathrm{H}_{2} \mathrm{O}: 60 \%{ }^{1} \mathrm{H}_{2} \mathrm{O}$ and $100 \%{ }^{1} \mathrm{H}_{2} \mathrm{O}$. Good scatter data was obtained for the high contrast condition of $100 \%{ }^{2} \mathrm{H}_{2} \mathrm{O}$ and $100 \%{ }^{1} \mathrm{H}_{2} \mathrm{O}$ and the pair distance distribution $P(R)$ functions obtained from this data are given in Figure 1 . In the attached reprint the $P(R)$ functions for the 146 bp core particle goes to zero at the maximum 
dimension in the particle of $12.0 \mathrm{~nm}$. As can be seen in Figure 1 for the 195 bp DNA particle in ${ }^{1} \mathrm{H}_{2} \mathrm{O}$ the $\mathrm{P}(\mathrm{R})$ function of the nucleosome goes to zero at $15.0 \mathrm{~nm}$ and for the particle in $100 \%{ }^{2} \mathrm{H}_{2} \mathrm{O}$ the $\mathrm{P}(\mathrm{R})$ function goest out to $13.5 \mathrm{~nm}$. Both of these results are consistent with the increased DNA length extending out from the nucleosome particle. These results demonstrate that the method we have proposed to study the effects of chemical modification on nucleoosme structure, particularly on the paths of the DNA entering and leaving the nucleosome, are viable. However, this study was not completed, again because of instabilities in the delivery of the proton beam to the spallation source. The statistics of the low contrast data at $65 \%{ }^{2} \mathrm{H}_{2} \mathrm{O}: 37 \%$ ${ }^{1} \mathrm{H}_{2} \mathrm{O}$ and $40 \%{ }^{2} \mathrm{H}_{2} \mathrm{O}: 60 \% 1$ were too poor to obtain reliable scatter functions and delivery of the beam was interrupted before the study could be completed. This study will have to be completed before we can address the effect of the variables associated with chromosome/functions on nucleosome and chromatin structure.

III) Location of the very lysine rich histone $\mathrm{H} 5$ on the nucleosome. The $195 \mathrm{bp}$ particle containing the histone octamer can be fully reformed into the nucleosome by the addition of a very lysine rich histone $\mathrm{H} 1, \mathrm{H}^{\circ}$ or $\mathrm{H} 5$. We have performed a neutron scatter experiment at LANSCE on 195 bp nucleosomes containing histone H5. Good scatter data was obtained for the high contrast conditions of the following four measurements: $100 \%{ }^{2} \mathrm{H}_{2} \mathrm{O} ; 75 \%$ ${ }^{2} \mathrm{H}_{2} \mathrm{O}: 25 \%{ }^{1} \mathrm{H}_{2} \mathrm{O} ; 25 \%{ }^{2} \mathrm{H}_{2} \mathrm{O}: 75 \%{ }^{1} \mathrm{H}_{2} \mathrm{O}$ and $100 \%{ }^{1} \mathrm{H}_{2} \mathrm{O}$. However, before all the background and blank neutron scatter could be obtained, the neutron beam was switched of for six months on October 1,1989 . The $\mathrm{P}(\mathrm{R})$ functions for the $100 \%{ }^{2} \mathrm{H}_{2} \mathrm{O}$ and $100 \%{ }^{1} \mathrm{H}_{2} \mathrm{O}$ from the $\mathrm{H} 5$ containing nucleosome are given in Figure 2. In both cases they show that the $P(R)$ function goes to zero at a smaller $R$ by about $0.5 \mathrm{~nm}$ compared to the control $195 \mathrm{bp}$ particle not containing histone H5. Again we have obtained interesting results but not a complete set of data.

\section{Proposed Experiments for $1989 / 90$}

We have shown that the Low Q Diffractometer (LQD) at LANSCE is capable of giving useful neutron scatter data on complex biological systems. This data is comparable to that obtained on DII at the Institute Laue Langevin, Grenoble but takes longer to obtain. Whereas a full contrast study can be obtained in 12 hours on D11 at the ILL it takes at least 60 hours on LQD at LANSCE. The reliability of the delivery of the neutron beam at LANSCE has also been a problem but this is improving. The three experiments that we carried out were not completed because of major interruptions in the beam delivery.

For $1989 / 90$ we propose:

i) to complete the three experiments described above.

ii) develop the biochemical procedures required to prepare fully defined nucleosomes and chromatin samples.

iii) determine the effects of histone hyperacetylation on nucleosome and chromatin structure.

\section{Appendix I}

Hyperactylation of Core Histones Does Not Cause Unfolding of Nucleosomes by Imai, B.S., Yau, P., Baldwin, J.P., Ibel, K., May, R.P. and Bradbury, E. M. (1986) J. Biol. Chem., 261, 8784-8792. 


\section{Papers}

1. Transcription factor IIIA induced binding of the Xenopus somatic 5S gene promoter by Schroth, G.P., ook, G.R., Bradbury, E.M. and Gottesfeld, J.M. (1989) Nature 270, 487488.

Work completed; Manuscripts completed or in preparation

2. Reconstitution of fully acetylated nucleosomes from defined systems by Marvin, K.M. and Bradbury, E.M. - manuscript completed.

3. Chromatosome positioning in reconstituted defined long chromatin by Meersseman, G., Pennings, S. and Bradbury, E.M. - manuscript completed.

4. "Neutron scatter studies of the shape of the histone octamer and trimmed histone octamer by Wood, M., Yau, P., Baldwin, J.P., Davie, J.R., Moudrianakis, E. and Bradbury, E.M. - manuscript almost completed. 

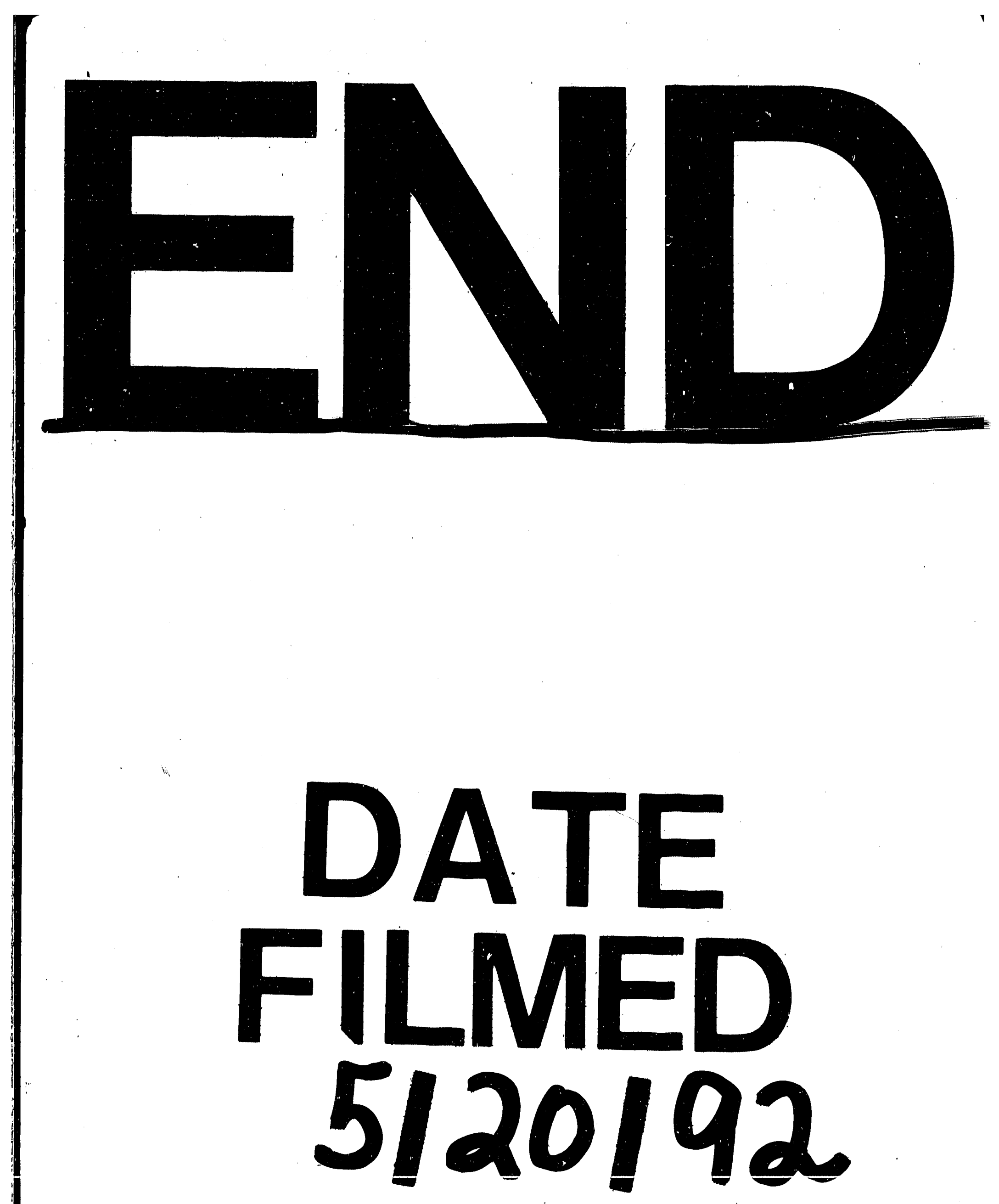


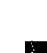

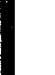

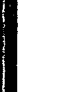

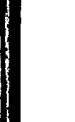
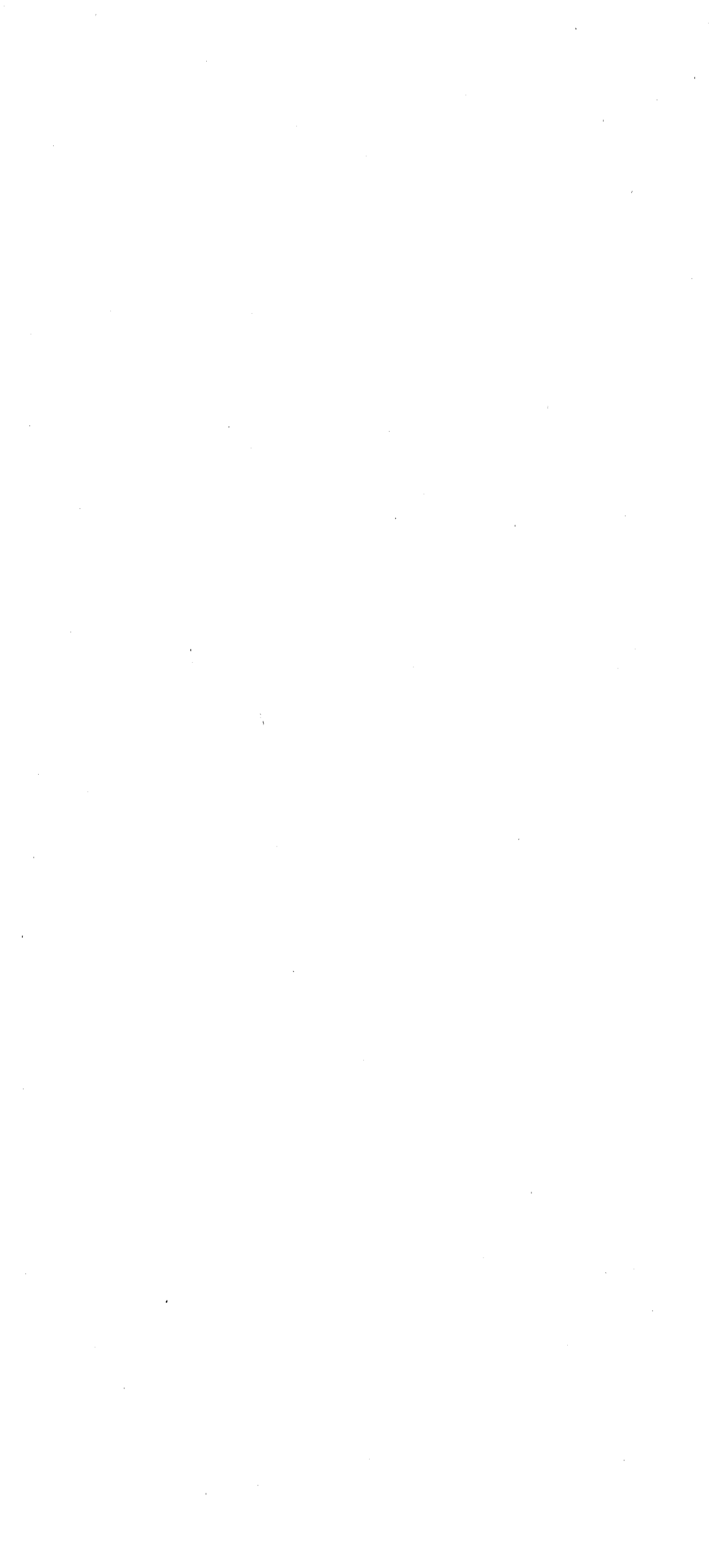